\title{
Construction and characterization of human oral mucosa equivalent using hyper-dry amniotic membrane as a matrix
}

\author{
Fangfang $\mathrm{Qi}^{\mathrm{a}}$, Toshiko Yoshida ${ }^{\mathrm{b}}$, Takeshi Koike ${ }^{\mathrm{c}}$, Hitoshi Aizawa ${ }^{\mathrm{a}}$, Tetsu Shimane ${ }^{\mathrm{a}}$, \\ Yinghui $\mathrm{Li}^{\mathrm{a}}$, Shinichi Yamada ${ }^{\mathrm{a}}$, Motonori Okabe ${ }^{\mathrm{b}}$, Toshio Nikaido ${ }^{\mathrm{b}}$, Hiroshi Kurita ${ }^{\mathrm{a}, *}$ \\ ${ }^{a}$ Department of Dentistry and Oral Surgery, Shinshu University School of Medicine, 3-1-1 Asahi, Matsumato 390-8621, Japan \\ bepartment of Regenerative Medicine, University of Toyama Graduate School of Medicine and Pharmaceutical Sciences, 2630 Sugitani, Toyama 930-0194, \\ Japan \\ ${ }^{\mathrm{C}}$ Department of Dentistry and Oral Surgery, Ina Central Hospital, 1313-1 Koshiroukubo, Ina, Nagano 396-8555, Japan
}

\section{A R T I C L E I N F O}

\section{Article history:}

Received 18 August 2015

Received in revised form 6 December 2015 Accepted 19 January 2016

\section{Keywords:}

Oral keratinocytes

Hyper-dry amniotic membrane

Matrix

Oral mucosal equivalent

Reconstruction

\begin{abstract}
A B S T R A C T
Background: Human amniotic membrane(HAM) as a graft material has been used in various fields. Hyperdry amniotic membrane (HD-AM) is a novel dried amniotic membrane that is easy to handle and can be preserved at room temperature without time limitation. The purpose of this study was to investigate the useful properties of HD-AM in reconstruction of the oral mucosa.

Methods: Human oral keratinocytes were isolated and seeded on HD-AM in serum-free culture system. Oral mucosa equivalent (OME) was developed and transplanted onto full-thickness wound on athymic mice. The wound healing was analyzed and the OME both before and after transplantation was analyzed with hematoxylin-eosin staining and immunohistochemicial staining for Cytokines 10 (CK10), Cytokines 16 (CK16), and Ivolucrin (IVL).

Results: Oral keratinocytes spread and proliferated well on HD-AM. Two weeks after air-lifting, OME had formed with good differentiation and morphology. We confirmed immunohistochemically that the expression of CK10 was positive in all suprabasal layers, as was CK16 in the upper layers, while IVL was present in all cell layers. Three weeks after transplantation to athymic mice, the newly generated tissue had survived well with the smallest contraction. The epithelial cells of newly generated tissue expressed CK10 throughout in all suprabasal layers, IVL was mainly in the granular layer, and CK16 positive cells were observed in all spinous layer and granular layer but were not expressed in the mouse skin, all of which were similar to native gingival mucosa.

Conclusions: The OME with HD-AM as a matrix revealed a good morphology and stable wound healing. This study demonstrates that HD-AM is a useful and feasible biomaterial for oral mucosa reconstruction.
\end{abstract}

(c) 2016 Elsevier Ltd. All rights reserved.

\section{Introduction}

In oral and maxillofacial surgery, biological tissue materials including tissue-engineered oral mucosa equivalent have been developed and used for repairing soft-tissue defects caused by trauma, surgery, etc. (Rouabhia \& Allaire, 2010; Terada, Izumi, \& Ohnuki, 2012). A variety of materials have been used as the platform for supporting cell attachment, proliferation and differentiation, promoting tissue regeneration and reconstruction, and facilitating integration of new tissue with the host (Atala, 2003; Gower \& Shea, 2013; Horst, Madduri, \& Milleret, 2013). As a biological tissue material, human amniotic membrane (AM) has

\footnotetext{
* Corresponding author.

E-mail address: hkurita@shinshu-u.ac.jp (H. Kurita).
}

low immunogenicity and has been widely used in clinical practice to date (Lo \& Pope, 2009; Shahdadfar, Haug, \& Pathak, 2012; Yang, Shirakata, \& Tokumaru, 2009). AM is a thin membrane in the inner side of the fetal placenta comprising three main layers: the epithelial layer, the thick basement layer and avascular stroma. As a basement membrane AM can promote tissue epithelialization (Shimazaki, Shinozaki, \& Tsubota, 1998; Meller \& Tseng, 1999) with non tumorigenicity and few ethical issues (Kamiya, Wang, Uchida, \& Amano, 2005; Robinson, McFadden, \& Barrentt, 2002).

Two types of AM, fresh AM and preserved AM, are usually used by researchers. Fresh AM retains the complete structure and cytokines and may have a slight antigenicity due to its cell component. Moreover, fresh AM must be used within $24 \mathrm{~h}$ (Akle, Adinolfi, \& Welsh, 1981; Lee \& Tseng, 1997). Thus, fresh AM has potential risks and is not so convenient for clinical application. Methods such as freeze-drying, cryopreservation and glycerol- 
preservation have been used to preserve AM (Prabhasawat \& Tesavibul, 2001; Wolbank, Hildner, \& Redl, 2009), but it has been reported that several growth factors and cytokines are absent from cryo-preserved and glycerol-preserved AM (Van Baare, Buitenwerf, \& Hoekstra, 1994). Although maintaining the intact structure and function of preserved AM is very important (Toda, Okabe, Yoshida, \& Nikaido, 2007), sterilization of AM is also essential to ensure a safe application, whereas glycerol is not considered to be a sterilizing agent (Von Versen-Honeynck, Steinfeld, \& Becker, 2008). As a novel dried-and-preserved-AM, hyper-dry amniotic membrane (HD-AM) partly overcame this problem by using farinfrared rays, microwaves, $\gamma$-irradiation sterilization, and low-airpressure drying (Okabe, Kitagawa, \& Yoshida, 2014). HD-AM not only keeps the tissue integrity structure similar to that of fresh AM, but also can be stored long-term at room temperature (Arai, Tsuno, \& Okabe, 2012). HD-AM can be easily and quickly cut into a suitable shape for application in any environment including the oral environment (Izumi Takacs \& Terashi, 1999; Tsuno, Arai, \& Sakai, 2014). Its usefulness as a wound-dressing material for surgical defects of the oral mucosa has been reported (Okabe et al., 2014).

AM is also considered an important potential source for scaffolding material. The special structure and biological viability of the AM makes it an ideal candidate for creating scaffolds used in tissue engineering (Nikenjad, Peirovi, \& Jorjani, 2008), but it has not yet been thoroughly investigated for use as a matrix for oral keratinocytes culture to reconstruct tissue defects. In this study, human oral keratinocytes were cultured on HD-AM and developed into stratified oral mucosa equivalents (OME). The OME was then fabricated and transplanted onto mice with full-thickness skin defects and the wound healing was evaluated. This study aimed to analyze the potential use of HD-AM-based OME in epithelial tissue reconstruction.

\section{Materials and methods}

This study protocol was approved by the Shinshu University Ethics committee, and was in accordance with the tenets of Declaration of Helsinki for research involving human subjects.

\subsection{Oral keratinocytes isolation and cultures}

Gingival mucosa was obtained from dental extraction patients with their informed consent. After the subcutaneous tissue was trimmed off, oral mucosa biopsies, each $4-6 \mathrm{~mm}^{2}$, were treated with $0.25 \%$ trypsin-EDTA solution (Wako, Osaka, Japan) overnight at $4{ }^{\circ} \mathrm{C}$. Enzyme activity was stopped by washing with trypsin neutralizing solution (TNS, Lonza, Walkersville, USA). The epithelial side of the mucosa was gently scraped with a forceps and scalpel to detach the keratinocytes. Subsequently, the resulting cell suspension was centrifuged at $1200 \mathrm{rpm}$ for $6 \mathrm{~min}$, and resuspended cells were cultured in serum-free KBM-Gold ${ }^{\mathrm{TM}}$ Basal
Medium supplemented with $\mathrm{KGM}^{\mathrm{TM}}-\mathrm{CD}$ single Quts ${ }^{\mathrm{TM}}$ (Lonza, Walkersville, USA) and incubated at $37^{\circ} \mathrm{C}$, in $5 \% \mathrm{CO}_{2}$ humidified air. Second-passage oral keratinocytes were used for the fabrication of OME.

\subsection{Production of tissue-engineered OME}

The HD-AM used in our experiment was provided by the University of Toyama (Toda et al., 2007). HD-AM was put on the bottom of a six-well culture plate of suitable size, and stabilized by overlying a resin buckle ring with inner diameter $1.5 \mathrm{~cm}$ (Fig. 1). Then HD-AM was moistened by immersion in PBS solution and incubated at $37^{\circ} \mathrm{C}, 5 \% \mathrm{CO}_{2}$ in humidified air for half an hour. Oral keratinocytes were seeded onto the HD-AM at a density of $1.25 \times 10^{5} / \mathrm{cm}^{2}$, which was found to be the optimal density for development of OME (Tsuno et al., 2014). The same density of oral keratinocytes was seeded on polycarbonate membrane (Millicell cell culture plate insert: MPI, Millipore Corp, Bedford, MA, USA) in a six-well culture plate as a control. Keratinocytes seeded on HD-AM and on MPI were all cultured while submerged for 4 days and then grown at an air-liquid interface for two weeks by the use of serumfree KBM-Gold ${ }^{\mathrm{TM}}$ medium containing calcium at a concentration of $1.8 \mathrm{mmol} / \mathrm{L}$. The medium was changed every 2 days. At the time point of $12 \mathrm{~h}, 4$ days and 12 days, the keratinocytes were confluent and multilayered on HD-AM and MPI, and photographs were taken with a phase-contrast microscope (CKX41, Olympus, Japan). On day 18 , samples of equivalents were taken for conventional histological and immunohistological examinations.

\subsection{Grafting of OME onto athymic mice}

Twenty-five male athymic mice (BALB/C, CLEA, Japan) aged 6-8 weeks were used for the experiment. All experimental protocols were approved by the Shinshu University Animal Care and Use committee.

Two types of OME - keratinocytes seeded on HD-AM and those seeded on MPI - were prepared for grafting. The mice were anesthetized by intraperitoneal injection of $6.48 \mathrm{mg} / \mathrm{mL}$ sodium pentobarbital at dose of $5 \mu \mathrm{L} / \mathrm{g}$ of body weight. Three squared fullthickness skin flaps that matched with OME were raised on the depilated dorsum of each mouse. The two types of OME of size $1 \times 1 \mathrm{~cm}^{2}$ were each grafted directly on the muscle tissue of the defect, and the remaining site was not covered to serve as a control. The graft sites were randomly changed between the mice. In order to improve the survival of the graft, all defects were covered with a silicone gel sheet $(0.09 \mathrm{~cm}$ thick, Silicone, Henderson, USA) and the skin flaps were returned and sutured into the original positions (Fig. 2). The skin flaps were surgically remove after 5-7 days. Three weeks after transplantation, grafted OME were harvested and excised together with surrounding tissue for histological and immunohistological examinations.
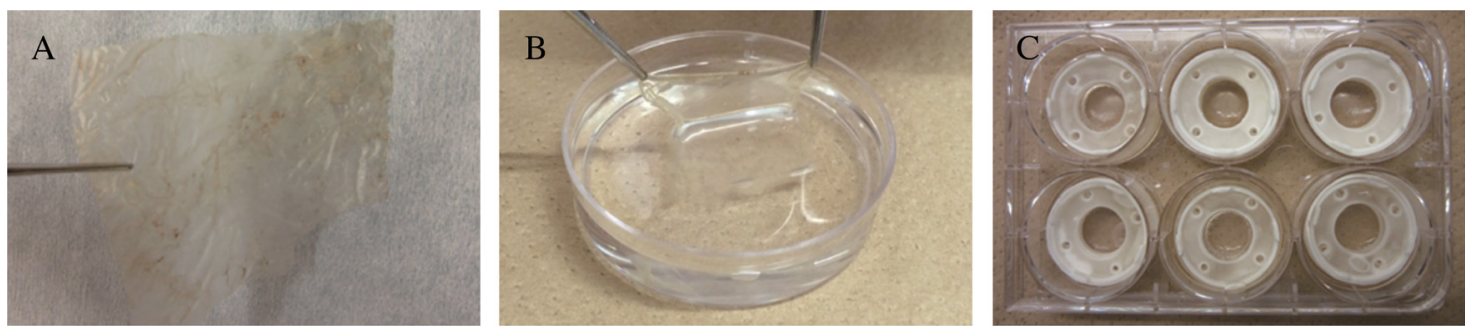

Fig. 1. Schematic illustration of culture procedure of keratinocytes seeded on hyper-dry amniotic membrane (HD-AM). (A) The appearances of HD-AM, (B) HD-AM rehydrated in PBS, (C) stabilized HD-AM by overlaying a resin buckle ring and culturing on a six-well culture plate. 

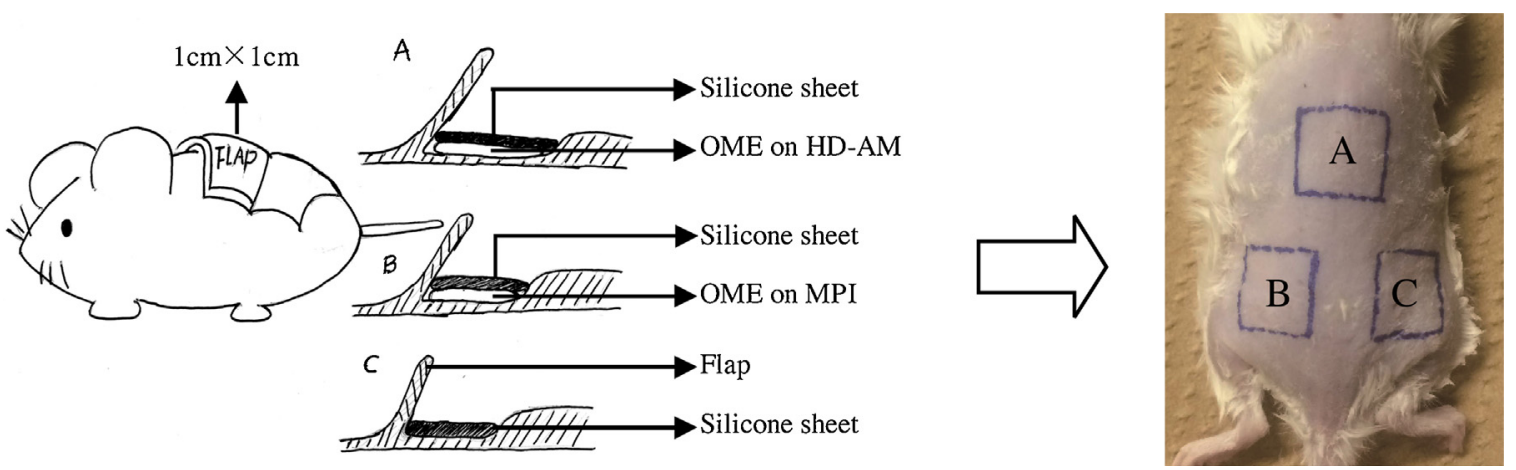

Fig. 2. Schematic illustration of grafting procedure. (A) OME on HD-AM group; (B) OME on millicell cell culture plate insert (MPI) group; (C) control group. The graft sites (AC) were randomly changed between the mice.

\subsection{Histological and immunohistological staining}

All the specimens were fixed with $10 \%$ neutral buffered formalin, embedded in paraffin and cut into $5-\mu \mathrm{m}$ sections. Samples of native human gingival and mouse back skin were used for comparison with derived OME. Sections were stained with either hematoxylin and eosin or used for immunostaining.

For immunostaining, the primary antibodies used in the study were rabbit monoclonal anti-CK10 (Funakoshi, Tokyo, Japan), mouse monoclonal anti-CK16 (Funakoshi, Tokyo, Japan), and mouse monoclonal anti-Ivolucrin (IVL, Sigma-Aldrich, Tokyo, Japan). After deparaffinization and hydration, the slices were fixed in $3 \%$ hydrogen peroxide formaldehyde solution for $30 \mathrm{~min}$ at room temperature to block endogenous peroxidase reactivity, and then incubated with primary antibodies with dilution of CK10 (1:200), CK16 (1:300), IVL (1:500) overnight at $4{ }^{\circ} \mathrm{C}$. After the sections were rinsed with Tris- $\mathrm{HCl}$ Buffer Saline (TBS), they were incubated with anti-rabbit/mouse secondary antibody at room temperature for $30 \mathrm{~min}$. Finally, after rinsing with TBS, the sections were treated with 3-3'-diaminobenzidine tetrahydrochloride (DAB) solution to visualize the reaction product. Sections were observed using a light microscope (Biorevo BZ-9000, Keyence, Osaka, Japan).

\subsection{Assessment of healing and contraction of the skin defect}

The sizes of skin defects created on the backs of the mice were measured and calculated before and three weeks after grafting. Each wound was photographed adjacent to a metric ruler for distance calibration and standardization. The wound area was calculated and quantified using the Image software in each photograph. The wound contraction rate was calculated according
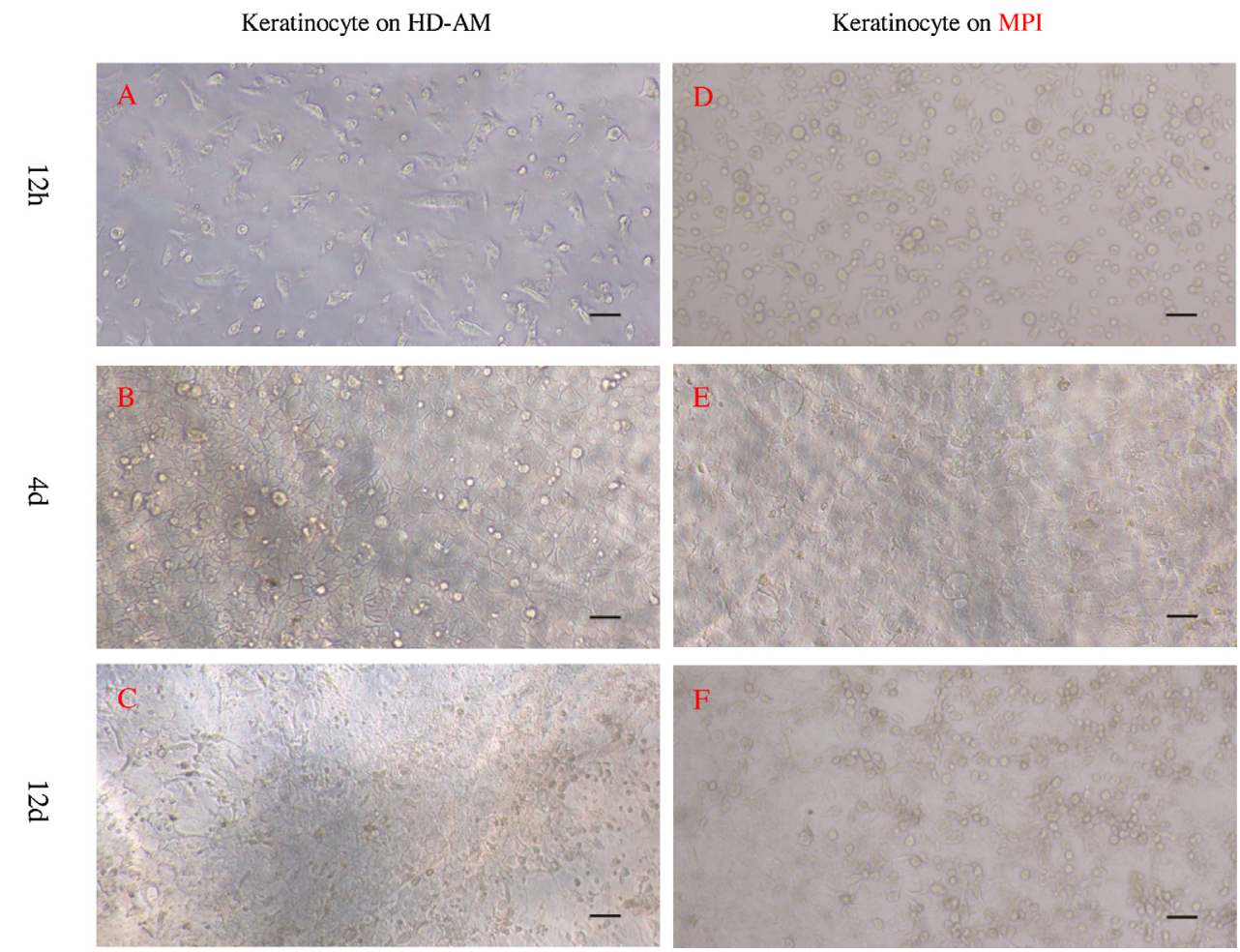

Fig. 3. Growth of keratinocytes seeding on HD-AM (A-C) and MPI (D-F). Twelve hours after seeding, the cells were attached and proliferating on HD-AM and MPI (A and D). Four days later, the cells reached confluence (B and E) and twelve days later, stratified layers had developed (C and F). Bars are $80 \mu \mathrm{m}$. 
to the following equation: wound contraction rate $=$ (1-re-epithelialization area/original wound area) $\times 100 \%$ (Haifei, Xingang, \& Shoucheng, 2014).

\subsection{Statistical analysis}

All data are presented as means \pm SD. Statistical analysis was performed using the two-tailed Student's $t$-test for comparisons of two groups (Graphpad Prism 6, Graphpad Software, California, USA). Values of $P<0.05$ were considered to indicate statistical significance.

\section{Results}

3.1. Growth of keratinocyte seeded on HD-AM and MPI (macroscopic findings)

Twelve hours after seeding oral keratinocytes on HD-AM and MPI, keratinocytes were attached to the HD-AM and MPI, and the morphology of keratinocyte was observed. In comparison with the small and round cells on MPI, most of the cells on HD-AM became larger and began to present a spindle shape. At day four, the cells reached confluence and were uniform in size and morphology on HD-AM, polygonal cells showed a pavement stone-like appearance. In contrast, cells on MPI appeared larger than those on HD-AM and were arranged irregularly. At day twelve, a stratified epithelium containing several layer of cells was present on HD-AM with compact structure and uniform thickness, while on MPI, the cultured epithelium had fewer cell layers and uneven surface (Fig. 3).

\subsection{Property of OMEs}

Two weeks after air-lifting, keratinocytes cultured on HD-AM were harvested and used to form the OME. The OME revealed good mechanical and handing properties. It had a yellowish appearance with uniform thickness. The generated epithelium had grown together with the HD-AM, and it could be transferred conveniently from the culture dish and trimmed to the required shape. However, the thinner epithelium on MPI often detached from MPI with a white appearance, making it difficult to transplant (Fig. 4).
3.3. Histological characteristics and immunohistochemical findings of $O M E$

Histologically, HD-AM tissue contained a thick basement membrane with individual epithelial cells (Fig. 5A). On the day eighteen, the keratinocytes on HD-AM derived OME mostly consisted of five-seven layers of cells with differentiated basal layer, spinous layer, granular layer and stratum corneum. Cuboids and small basal cells were compactly aligned on HD-AM, and sporadic nuclei were often seen in the well-structured stratum corneum (Fig. 5B). The keratinocytes cultured on MPI showed a sparse structure with thin layers that could easily detach form the MPI (Fig. 5C).

As newly regenerated tissue, it is important to confirm the feasibility of using OME as an implant to regenerate functional mucosa. As can be seen in Fig. 6, CK10, which is regarded as a marker of keratinization, was present on all the super basal layers including the stratum corneum (Fig 5D). IVL is a useful marker for terminal differentiation of keratinocytes, and IVL positive cells were spread throughout almost all the epithelial layers (Fig. 5E). Furthermore, CK16 stained cells were expressed in the upper layers of tissue, similarly to gingival mucosa tissue (Fig. 5F).

\subsection{Analysis of wound healing (shrinkage)}

Three weeks after grafting, all wounds were completely healed with no inflammatory or necrotic problems. Although newlygenerated mucosa had completely integrated with the surrounding mouse epidermis, a clear boundary line was still seen between them because no hair follicles were observed on the graft site. Here we focus on the re-epithelialization area of the wounds. In the HDAM group, the newly regenerated epithelialization was seen in the center of the original wound with a square shape similar to the original defect. In the other two groups this was obviously shrunken and healing into an irregular shape (Fig. 6). The calculated constriction rates were as follows: HD-AM group $63.90 \pm 0.87 \mathrm{~mm}^{2}$; MPI group $77.13 \pm 5.75 \mathrm{~mm}^{2}$; control group $87.02 \pm 2.27 \mathrm{~mm}^{2}$. The HD-AM group showed significantly less constriction compared with either the MPI group $(P<0.05)$ or control group $(P<0.001)$ (Fig, 7).
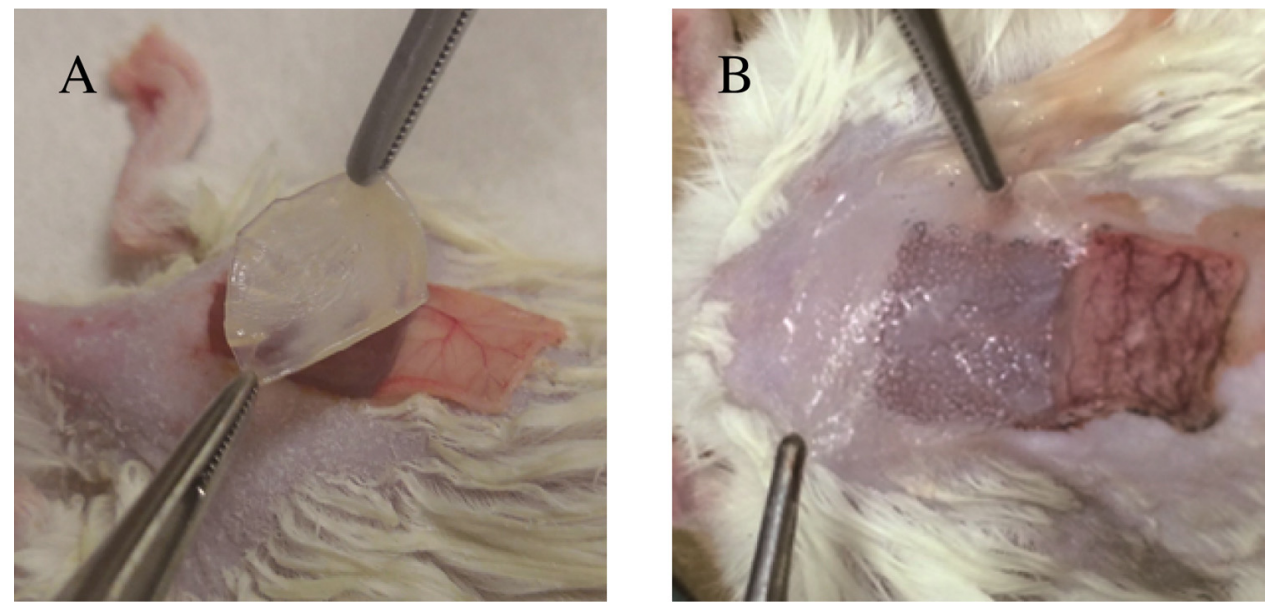

Fig. 4. Property of oral mucosa equivalent (OME). OME on hyper-dry amniotic membrane with uniform thickness revealed good mechanical and handing properties (A), while OME on Millicell cell culture plate insert was thinner and difficult to transplanting (B). 

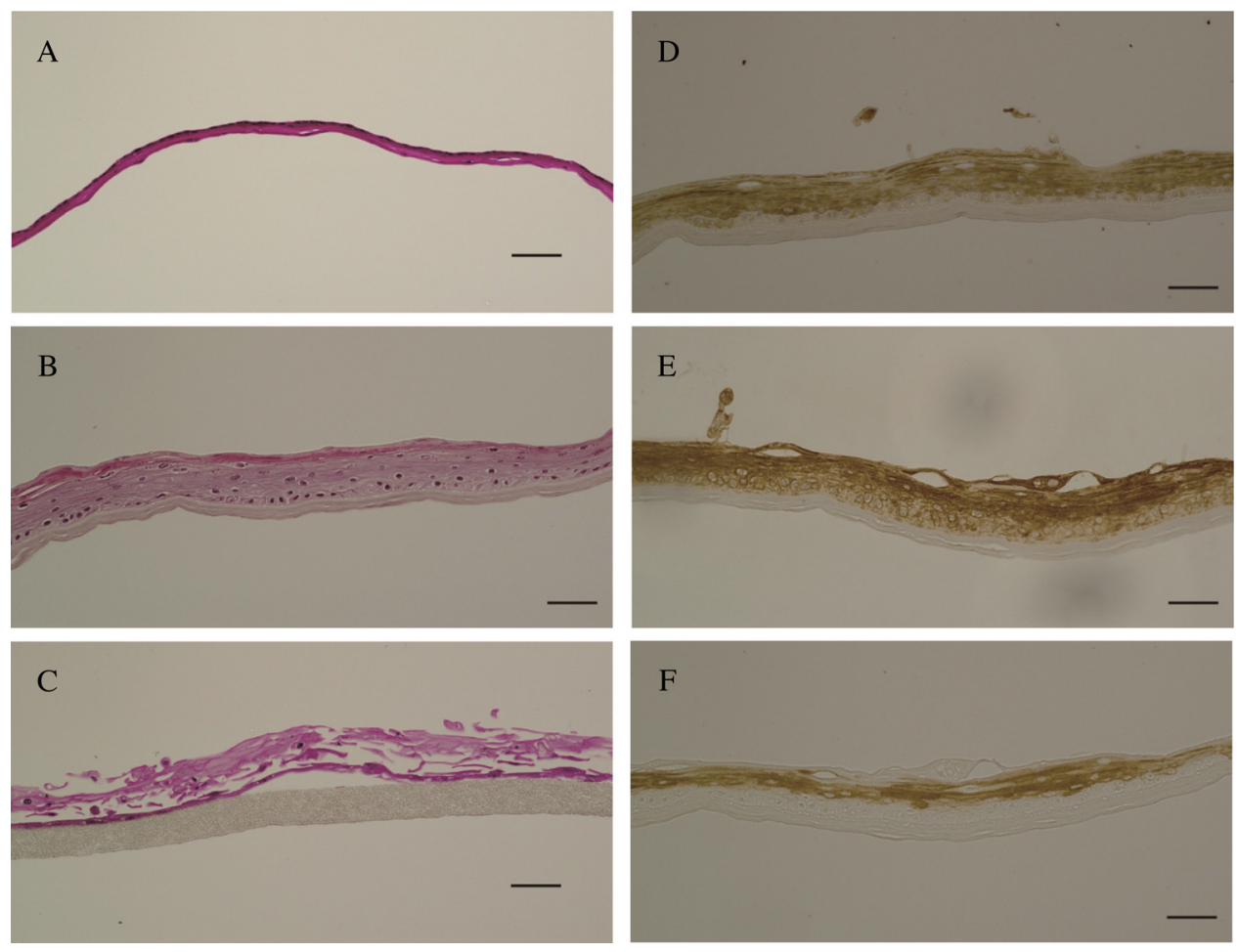

Fig. 5. Histological characteristics of biological tissue materials used in the study. H-E staining of hyper-dry amniotic membrane (HD-AM) (A), oral mucosa equivalents (OME) cultured on HD-AM (B), OME cultured on millicell cell culture plate insert (C). Immunohistochemical expression of CK10 (D), IVL (E) and CK16 (F) in OME on HD-AM. Scale bars are $60 \mu \mathrm{m}$; original magnification $\times 20$.

\subsection{Histological assessment of the grafted OMEs}

Three weeks after OME grafting, the newly generated tissue had grown well on the dorsum of athymic mice in the HD-AM group. Although the newly generated mucosa had completely integrated with the surrounding mouse epidermis, a clear boundary line was still seen between them because the mucosa had more layers of cells than the mouse epidermis and, especially, no hair follicles were observed on the graft site. These findings suggested that OME could grow into human mucosa without being rejected or degraded by mouse tissue (Fig. 8). As shown by histological examination, HD-AM and MPI were completely degraded without inflammation. The epithelium adhering to the connective tissue was well-organized, containing a compactly aligned basal layer, spinous layer, granular layer and stratum corneum.

In the results of the immunohistological staining, CK10 and IVL, which are implicated as differentiation markers, were expressed in the skin and oral mucosa. In generated tissue, CK10 positive cells were spread throughout all the superbasal layers (Fig. 9A-b) and IVL was mainly seen in the granular layer (Fig. 9A-c); all of this was similar to the native oral gingiva. CK16 is a marker expressed constitutively in palmar and plantar epidermis, buccal mucosa, gingival and tongue, but not in the skin (Gasparoni, Fonzi, \& Schneider, 2004). CK16 immunopositive cells were observed in all spinous layer and granular layers (Fig. 9A-d) but were not expressed in mouse skin or in the MPI group (Fig. 9B-d) and control group (Fig. 9C-d). The overall results thus indicated that the newly generated tissue was human origin.

\section{Discussion}

With the development of tissue engineering, oral mucosa equivalent (OME) has been developed and applied for soft tissue defects. It is well known that the scaffold material is an important factor in oral mucosa tissue engineering. Using AM as a natural regenerative biomaterial, the scope of clinical applications has
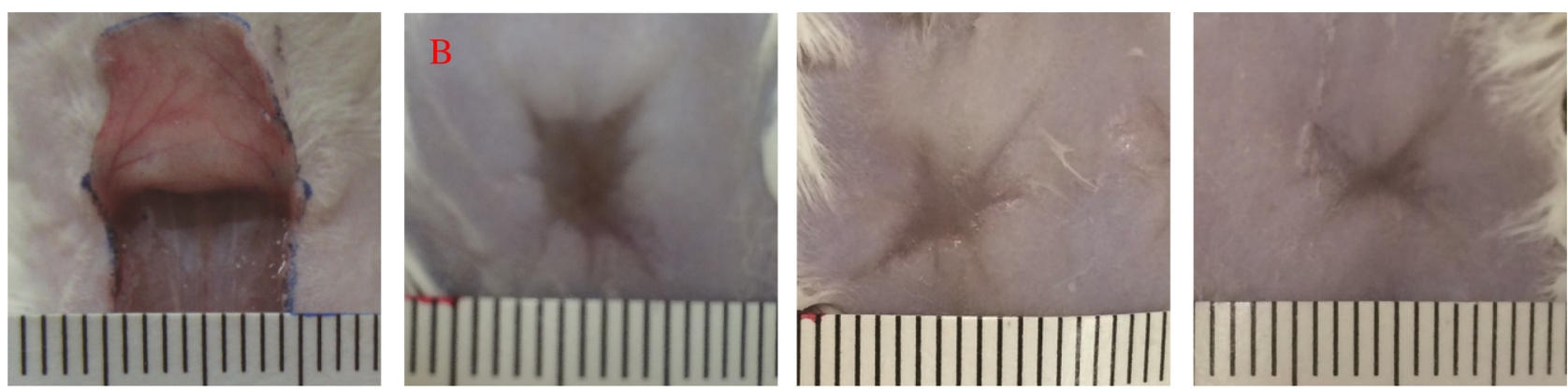

Fig. 6. Macroscopic appearance of wound healing. Skin defect before transplantation (A), three weeks after transplantation, wound healing with oral mucosa equivalents (OME) cultured on HD-AM (B), OME cultured on MPI (C), and no graft (D). 


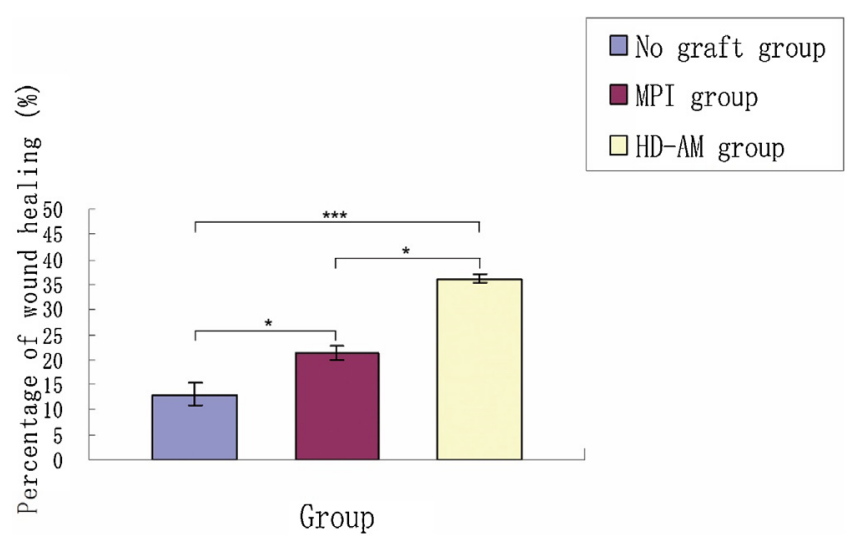

Fig. 7. Comparison of the wound contraction rate among defects covered with oral mucosa equivalents (OME) cultured on HD-AM, OME cultured on MPI, and no graft (Student's $t$-test; ${ }^{*} P<0.05,{ }^{* * *} P<0.001, n=25$ ).

been increasing in recent years (Jin, Park, \& Choi, 2007; Rohaina, Then, \& Ng, 2014). Based on such studies, for the first time we used a novel dried AM named HD-AM as culture substrate to expand oral keratinocytes in order to fabricate OME, and grafted OME to full-thickness mouse skin defects.

It is well know that using a feeder layer or FBS can increase the risk of contamination by unknown viruses or transfer exogenous components. To avoid this risk, we established serum-free culture conditions to develop OME (Izumi, Terashi, \& Marcelo, 2000). In order to successfully fabricate OME, selecting a suitable material as a matrix to support important functions including differentiation, proliferation and migration of oral keratinocytes during development is critical. Some studies used synthesized polymers to form skin equivalent because of their mechanical properties and rate of degradation (Horst et al., 2013; Zhang, He, \& Bharadwaj, 2009). MPI is a product used in tissue culture plate well inserts that contributes to the cells adhering and proliferating, and it is used as a scaffold for culture cell sheets (Koike, Yasuo, \& Shimane, 2011). In this study, by direct comparison with cultured keratinocytes on MPI, we found that keratinocytes spread and proliferated well on HD-AM, Three weeks after culturing, keratinocytes had grown and infiltrated onto HD-AM, and had derived several layers with good differentiation and good morphology (Fig. 5). HD-AM-formed OME resisted shearing and fit closely with mouse subcutaneous tissue with no bubbles while the MPI-formed OME readily detached form MPI and was easily destroyed by tweezers. This indicated that the biocompatibility and mechanical properties of AM are key benefits compared with other materials. Some studies have reported that AM contains natural structural and functional proteins such as collagen I, III, IV, V, VII, laminin and vimentin, all of which dynamically impact on the keratinocytes, migration, attachment and proliferation (Kikkawa, Umeda, \& Miyazaki, 1994; Koizumi, Inatomi, \& Sotozono, 2000). In addition, HD-AM preserved the
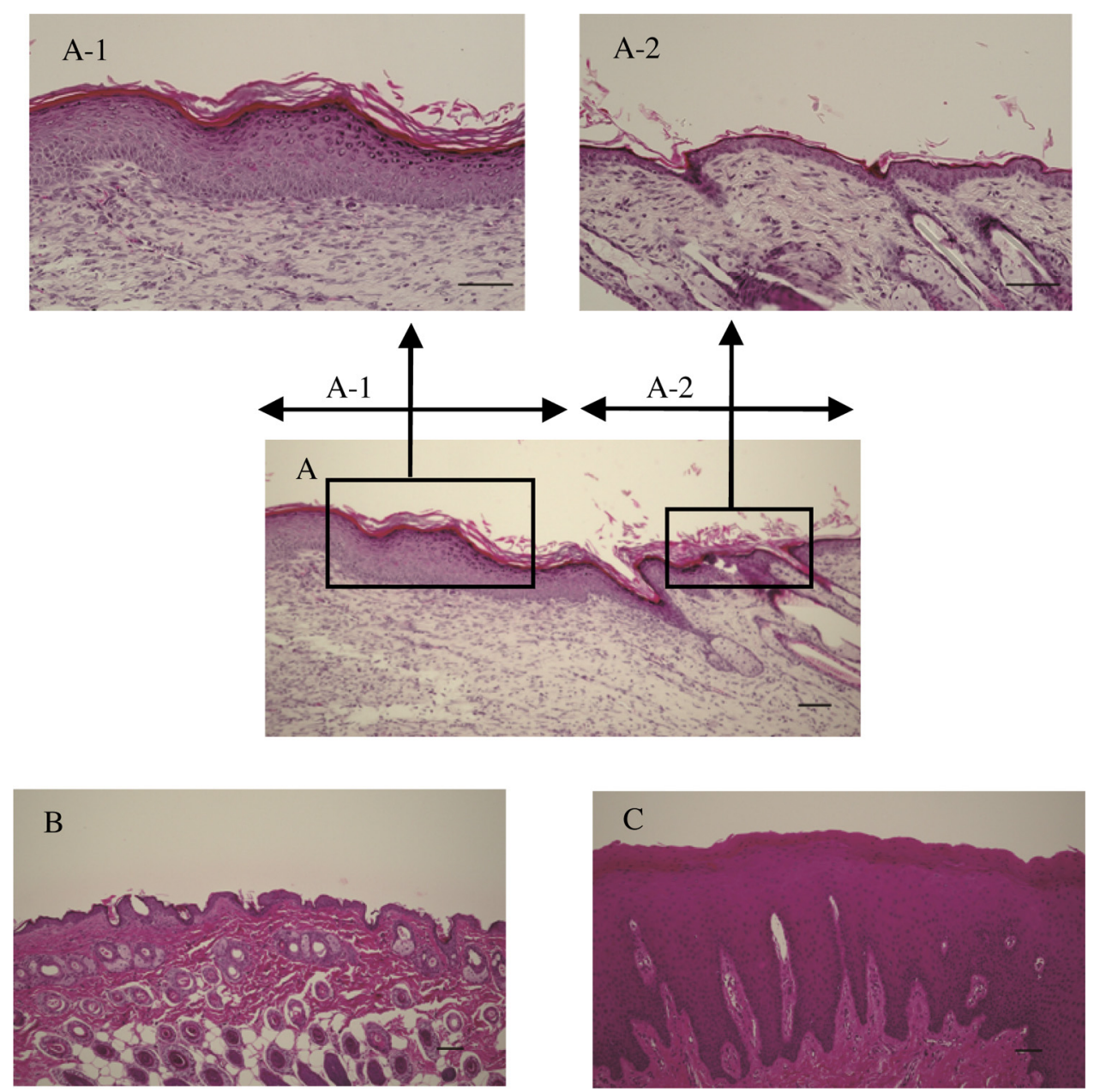

Fig. 8. Structural and morphology of OME cultured on HD-AM grafted onto a mouse skin defect. (H-E staining) the grafted site (A); the left half is the graft area (A-1), and the right half is the surrounding mouse epidermis (A-2). Normal human gingiva (B) and mouse skin (C). Scale bars are $80 \mu \mathrm{m}$; original magnification $\times 10$, A-1 and A- 2 are 20 magnifications $(\times 20)$. 

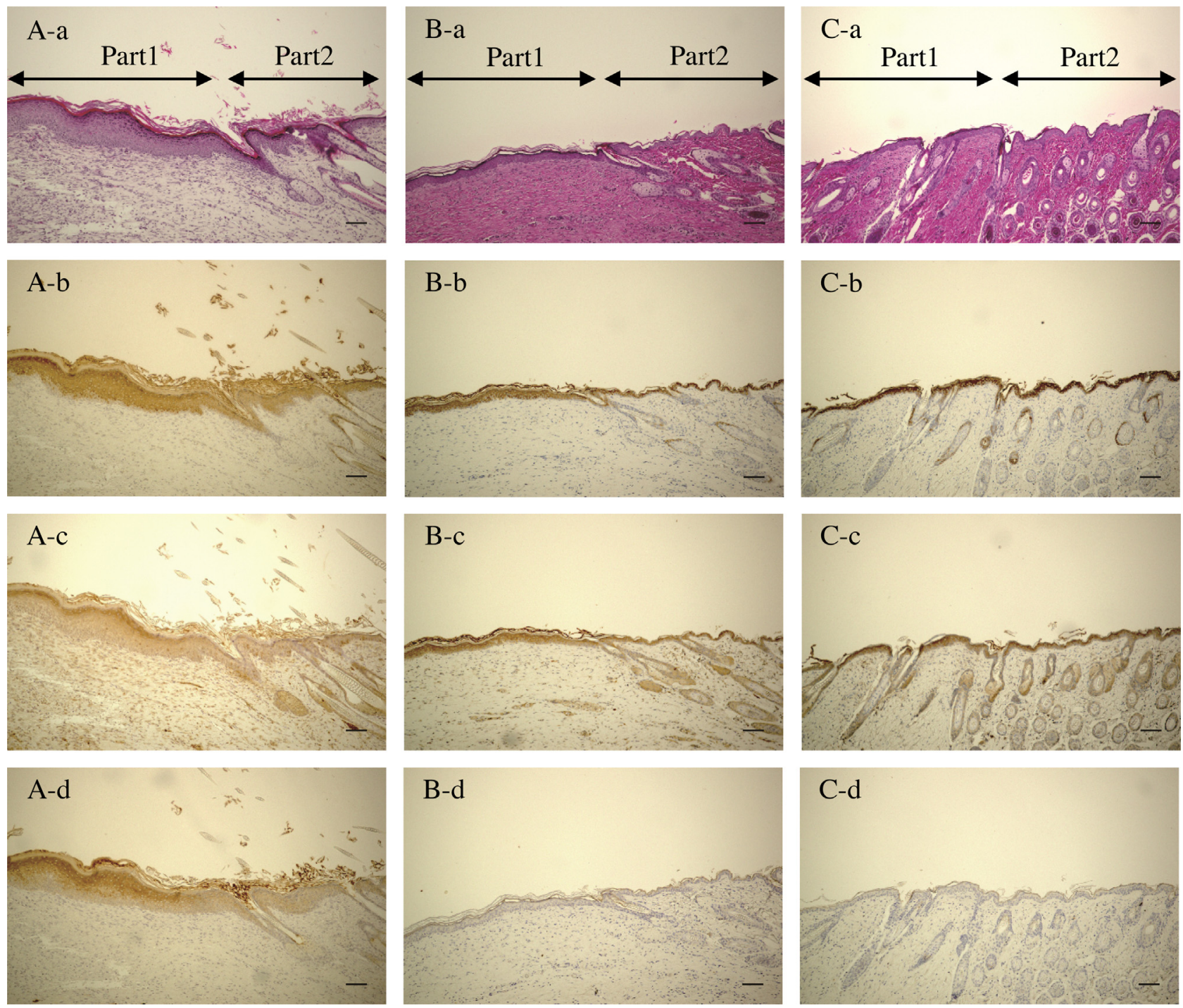

Fig. 9. Comparison of histological and immunohistological findings between skin defects covered with different materials (A: OME on HD-AM, B: OME on MPI, and C: control (no material)). H-E staining (A-a, B-a, C-a) and immunohistochemical expression of CK10 (A-b, B-b, C-b), IVL (A-c, B-c, C-c), and CK16 (A-d, B-d, C-d). Part 1 is the graft area; part 2 is the surrounding mice epidermis. Scale bars are $80 \mu \mathrm{m}$; original magnification $\times 10$.

mechanical behavior of AM; beside permeability, stability, and elasticity, also the effects of cell activity and differentiation (Kesting, Wolff, \& Mucke, 2009; Mamede, Carvalho, \& Abrantes, 2012). The results presented here confirmed that HD-AM not only retained the integrity structure and function of fresh AM, but also had a certain stiffness to withstand keratinocytes adherence and recover its shape. The permeability of HD-AM also can effectively deliver nutrients from the culture medium to the cells.

In the process of transplantation, in order to avoid contamination by mouse cells and to enhance survival of the graft, we fabricated a skin flap instead of using the traditional method using a full-thickness circular wound (Ahn, Lee, \& Hwang, 2006; Ji \& Xiao, 2011). Five-seven days after graft survival, the skin flap was removed. In addition, we covered the wound with a silicon gel sheet which is not only a useful dressing material but also can protect effectively against contamination by mouse cells. Histological studies showed that by three weeks after grafting, the layers of cells in the newly-generated epithelium were more significant than in the mouse epidermis and were without hair follicle. This result confirmed that there was no invasion of the implant by mouse cells, and this method may have been pivotal to the success of the transplant.
Three weeks after grafting, all the wounds had healed completely, the newly generated epithelium in the HD-AM group looked soft, flat, and was approximately square in the center of the wound. Statistically less shrinkage was observed in the HD-AM group than in the MPI group and control group. It is well known that, compared with human oral mucosa, mouse skin epithelium is much thinner and in loose contact with connective tissue. This might impact the area of newly generated epithelium. Some researchers have argued that HD-AM is not able to completely inhibit wound contraction (Loeffelbein, Baumann, \& Stoeckelhuber, 2012), but the incidence of infection and degree of contraction are related to whether a wound is covered with biological material or an artificial one (Tsuno et al., 2014). Our findings indicated that, on the one hand, as a biological material, HD-AM could supply sufficient mechanical stability to support tissue cell ingrowth and complete remodeling before it is biodegraded (Amemiya, Nakamura, \& Yamamoto, 2010). In this study, three weeks after transplantation, the newly generated mucosa had integrated with subcutaneous tissue, and the HD-AM had biodegraded with no inflammation. Moreover, the antimicrobial effect of HD-AM and its good histocompatibility all reduced the risk of inflammation and infection. When drying HD-AM, the 
boiling temperature could be decreased under low-air pressure conditions, which would further decrease the degradation of tissue proteins. High levels of TGF-a, EGF, KGF, HGF, bFGF, KGFR and HGFR were all expressed in AM (Koike et al., 2011), all these growth factors play important roles in promoting tissue re-epithelization and reconstruction and reduce the shrinkage during wound healing (Zhang et al., 2009).

To determine the tissue differentiation and structural features of OME, we examined the cytokeratin expression by immunocytochemistry. The differentiation markers CK10 and IVL are abundantly expressed in epidermis and oral mucosa. It is noteworthy in our results that CK10 positive cells were expressed in all the suprabasal layers in OME both before and after grafting. IVL positive cells were expressed throughout the entire epithelial layer in OME before grafting, while after grafting, IVL positive cells were expressed mainly in the granular layer in newly generated epithelium. Izumi et al. (2000). Showed that, CK10 positive cells were mainly restricted to the uppermost keratinized epidermis of 18 days cultured equivalent, but were not found on 4-day and 11day cultured equivalents. This suggested that oral keratinocytes were proliferating and differentiating well on HD-AM, and after three weeks it formed an ideal OME for grafting. Investigators have shown that IVL is cross linked into the cornified envelope of mucosa keratinocyte terminal differentiation; it is more responsive to the tissue differentiation stage reached by kerationcytes, while CK10 is responsive to the early kerationcytes differentiation (Alaminos, Garzon, \& Sanchez-Quevedo, 2007; Gasparoni et al., 2004). Here IVL as the only envelop precursor showed a major difference between OME before and after grafting. Before grafting, keratinocytes were highly proliferated and differentiated, but afterwards a mature oral mucosal structure and characteristics were observed.

Keratins are a family of related proteins that are predominantly cytoskeleton proteins in all epithelial layers, but keratin expression varies among tissues (Presland \& Dale, 2000), so we can distinguish tissues not only by morphology, but also by the expression of structural proteins. The epidermis of the skin and the oral mucosa are both highly specialized stratified epithelia, but because they are in different environments, the epithelial cells (keratinocytes) undergo a program of terminal differentiation expressing specific structures proteins. CK16 is a structural protein normally expressed in stratified epithelia including the palmar and plantar epidermis, nail bed, and the oral mucosa but CK16 is not normally expressed in the epidermis (Dale, Salonen, \& Jones, 1990). In the results of this study, three weeks after grafting of OME on HD-AM, CK16 positive stained cells were expressed on all spinous layer and granular layers in the re-epithelialization area, but were not expressed on the mouse skin. This result proved that newly generated epithelium retained the characteristics of human oral mucosa rather than the characteristics of mouse skin. Compared with OME on MPI, CK16 was not expressed in either the graft area or the surrounding mice epidermis. Possible reasons for this are that in the process of the production of OME, the oral keratinocytes do not grow well and get readily detached from MPI, or in the process of grafting of OME onto athymic mice, the oral keratinocytes became contaminated with mouse cells. To the best of our knowledge, this is the first study using CK16 to distinguish the re-epithelialization tissue from mouse skin.

In conclusion, HD-AM as a matrix-derived oral mucosal equivalent not only offered a morphology and structure similar to native tissue but also promoted healing and long-term stability of the closed wound. All our findings indicate that HD-AM is a useful and feasible biomaterial for oral mucosa reconstruction. We would encourage and support the use of HD-AM in the field of tissue engineering.

\section{Conflict of interest}

None.

\section{References}

Ahn, K. M., Lee, J. H., \& Hwang, S. J. (2006). Fabrication of myomucosal flap using tissue-engineered bioartificial mucosa constructed with oral keratinocytes cultured on amniotic membrane. Artificial Organs, 30(6), 411-423.

Akle, C. A., Adinolfi, M., \& Welsh, K. I. (1981). Immunogenicity of human amniotic epithelial cells after transplantation into volunteers. Lancet, 2(8254), 10031005.

Alaminos, M., Garzon, I., \& Sanchez-Quevedo, M. C. (2007). Time-course study of histological and genetic of differentiation in human engineered oral mucosa. Journal of Tissue Engineering and Regenerative Medicine, 1(5), 50-359.

Amemiya, T., Nakamura, T., \& Yamamoto, T. (2010). Immunohistochemical study of oral epithelial sheets cultured on amniotic membrane for oral mucosal reconstruction. Bio-Medical Materials Engineering, 20(1), 37-45.

Arai, N., Tsuno, H., \& Okabe, M. (2012). Clinical application of a hyperdry amniotic membrane on surgical defects of the oral mucosa. Journal of Oral and Maxillofacial Surgery, 70(9), 2221-2228.

Atala, A. (2003). Tissue engineering, stem cells and cloning for the regeneration of urologic organs. Clinics in Plastic Surgery, 30(4), 649-667.

Dale, B. A., Salonen, J., \& Jones, A. H. (1990). New approaches and concepts in the study of differentiation of oral epithelia. Critical Reviews in Oral Biology $\mathcal{E}^{\prime}$ Medicine, 1(3), 167-190.

Gasparoni, A., Fonzi, L., \& Schneider, G. B. (2004). Comparison of differentiation markers between normal and two squamous cell carcinoma cell lines in culture. Archives of Oral Biology, 49(8), 653-664.

Gower, R. M., \& Shea, L. D. (2013). Biomaterial scaffolds for controlled, localized gene delivery of regenerative factors. Advances in Wound Care, 2(3), 100-106.

Haifei, S., Xingang, W., \& Shoucheng, W. (2014). The effect of collagen-chitosan porous scaffold thickness on dermal regeneration in a one stage grafting procedure. Journal of the Mechanical Behavior of Biomedical Materials, 29 $114-125$.

Horst, M., Madduri, S., \& Milleret, V. (2013). A bilayered hybrid microfibrous PLGAacellular matrix scaffold for hollow organ tissue engineering. Biomaterials, 34 (5), 1537-1545.

Izumi Takacs, G., \& Terashi, H. (1999). Ex vivo development of a composite human oral mucosal equivalent. Journal of Oral and Maxillofacial Surgery, 57(5), 571-577.

Izumi, K., Terashi, H., \& Marcelo, C. L. (2000). Development and characterization of a tissue-engineered human oral mucosa equivalent produced in a serum-free culture system. Journal of Dental Research, 79(3), 798-805.

Ji, S. Z., \& Xiao, S. C. (2011). An epidermal stem cells niche microenvironment created by engineered human amniotic membrane. Biomaterials, 32(31), 7801-7811.

Jin, C. N., Park, S. R., \& Choi, B. H. (2007). Human amniotic membrane as a delivery matrix for articular cartilage repair. Tissue Engineering, 13(4), 693-702.

Kamiya, K., Wang, M., Uchida, S., \& Amano, S. (2005). Topic application of culture supernatant from human amniotic epithelial cells suppresses inflammatory reactions in cornea. Experimental Eye Research, 80(5), 671-679.

Kesting, M. R., Wolff, K. D., \& Mucke, T. (2009). Abioartificial patch from multilayered human amniotic membrane-in vivo investigations in a rat model. Journal of Biomedical Materials Research B, 90(2), 930-938.

Kikkawa, Y., Umeda, M., \& Miyazaki, K. (1994). Marked stimulation of cell adhesion and motility by ladsin, a laminin-like scatter factor. Journal of Biochemistry, 116 (4), 862-869.

Koike, T., Yasuo, M., \& Shimane, T. (2011). Cultured epithelial grafting using human amniotic amniotic membrane: the potential for using human amniotic epithelial cells as a cultured oral epithelium sheet. Archives of Oral Biology, 56 (10), 1170-1176.

Koizumi, N. J., Inatomi, T. J., \& Sotozono, C. J. (2000). Growth factor m RNA and protein in preserved human amniotic membrane. Current Eye Research, 20(3), 173-177.

Lee, S. H., \& Tseng, S. C. (1997). Amniotic membrane transplantation for persistent epithelial defects with ulceration. American Journal of Ophthalmology, 123(3), 303-312.

Lo, V., \& Pope, E. (2009). Amniotic membrane use in dermatology. International Journal of Dermatology, 48(9), 935-940.

Loeffelbein, D. J., Baumann, C., \& Stoeckelhuber, M. (2012). Amniotic membrane as part of a skin substitute for full-thickness wounds: an experimental evaluation in a porcine model. Journal of Biomedical Materials Research, 100(5), 1245-1256.

Mamede, A. C., Carvalho, M. J., \& Abrantes, A. M. (2012). Amniotic membrane: from structure and functions to clinical applications. Cell and Tissue Research, 394(2), 447-458.

Meller, D., \& Tseng, S. C. (1999). Conjunctival epithelial cell differentiation on amniotic membrane. Investigative Ophthalmology E Visual Science, 40(5), $878-886$.

Nikenjad, H., Peirovi, H., \& Jorjani, M. (2008). Properties of the amniotic membrane for potential use in the tissue engineering. European Cells E' Materials, 29(15), $88-99$

Okabe, M., Kitagawa, K., \& Yoshida, T. (2014). Hyperdry human amniotic membrane is useful material for tissue engineering: physical, morphological properties, and safety as the new biological material. Journal of Biomedical Materials Research A, 102(3), 862-870. 
Prabhasawat, P., \& Tesavibul, N. (2001). Preserved amniotic membrane transplantation for conjunctival surface reconstruction. Cell and Tissue Banking, 2(1), 31-39.

Presland, R. B., \& Dale, B. A. (2000). Epithelial structural proteins of the skin and ora cavity: function in health and disease. Critical Reviews in Oral Biology \& Medicine, 11(4), 383-408.

Robinson, W. P., McFadden, D. E., \& Barrentt, I. J. (2002). Origin of amnion and implication for evaluation of the fetal genotype in cases of mosaicism. Prenatal Diagnosis, 22(12), 1076-1085.

Rohaina, C. M., Then, K. Y., \& Ng, A. M. (2014). Reconstruction of limbal stem cell deficient corneal surface with induced human bone marrow mesenchymal stem cells on amniotic membrane. Translational Research, 163(3), 200-210.

Rouabhia, M., \& Allaire, P. (2010). Gingival mucosa regeneration in athymic mice using in vitro engineered human oral mucosa. Biomaterials, 31(22), 5798-5804.

Shahdadfar, A., Haug, K., \& Pathak, M. (2012). Ex vivo expanded autologous limbal epithelial cells on amniotic membrane using a culture medium with human serum as single supplement. Experimental Eye Research, 97(1), 1-9.

Shimazaki, J., Shinozaki, N., \& Tsubota, K. (1998). Transplantation of amniotic membrane and limbal autograft for patients with recurrent pterygium associated with symblepharon. British Journal of Ophthalmology, 82(3), 235-240.

Terada, M., Izumi, K., \& Ohnuki, H. (2012). Construction and characterization of a tissue-engineered oral mucosa equivalent based on a chitosan-fish scale collagen composite. Journal of Biomedical Materials Research, 100(7), 1792-1802.
Toda, A., Okabe, M., Yoshida, T., \& Nikaido, T. (2007). The potential of amniotic membrane/amnion-derived cells for regeneration of various tissues. Journal of Pharmacological Sciences, 105(3), 215-228.

Tsuno, T., Arai, N., \& Sakai, C. (2014). Intraoral application of hyperdry amniotic membrane to surgically exposed bone surface. Oral Surgery, Oral Medicine, Oral Pathology, Oral Radiology, 117(2), 83-87.

Van Baare, J., Buitenwerf, J., \& Hoekstra, M. J. (1994). Virucidal effect of glycerol as used in donor skin preservation. Burns, 20(1), 77-80.

Von Versen-Honeynck, F., Steinfeld, A. P., \& Becker, J. (2008). Sterilization and preservation influence the biophysical properties of human amnion grafts. Biologicals, 36(4), 248-255.

Wolbank, S., Hildner, F., \& Redl, H. (2009). Impact of human amniotic membrane preparation on release of angiogenic factors. Journal of Tissue Engineering and Regenerative Medicine, 3(8), 651-654.

Yang, L., Shirakata, Y., \& Tokumaru, S. (2009). Living skin equivalents constructed using human amnions as a matrix. Journal of Dermatological Science, 56(3), 188-195.

Zhang, Y., He, Y., \& Bharadwaj, S. (2009). Tissue-specific extracellular matrix coatings for the promotion of cell proliferation and maintenance of cell phenotype. Biomaterials, 30(23-24), 4021-4028. 